\title{
Haptic study of three-dimensional objects activates extrastriate visual areas
}

\author{
Thomas W. James, G. Keith Humphrey, Joseph S. Gati, Philip Servos, \\ Ravi S. Menon, Melvyn A. Goodale* \\ CIHR Group for Action and Perception, Psychology Department, The University of Western Ontario, London, Ont., Canada N6A 5C2
}

Received 5 March 2001; received in revised form 13 December 2001; accepted 20 December 2001

\begin{abstract}
In humans and many other primates, the visual system plays the major role in object recognition. But objects can also be recognized through haptic exploration, which uses our sense of touch. Nonetheless, it has been argued that the haptic system makes use of 'visual' processing to construct a representation of the object. To investigate possible interactions between the visual and haptic systems, we used functional magnetic resonance imaging to measure the effects of cross-modal haptic-to-visual priming on brain activation. Subjects studied three-dimensional novel clay objects either visually or haptically before entering the scanner. During scanning, subjects viewed visually primed, haptically primed, and non-primed objects. They also haptically explored non-primed objects. Visual and haptic exploration of non-primed objects produced significant activation in several brain regions, and produced overlapping activation in the middle occipital area (MO). Viewing visually and haptically primed objects produced more activation than viewing non-primed objects in both area MO and the lateral occipital area (LO). In summary, haptic exploration of novel three-dimensional objects produced activation, not only in somatosensory cortex, but also in areas of the occipital cortex associated with visual processing. Furthermore, previous haptic experience with these objects enhanced activation in visual areas when these same objects were subsequently viewed. Taken together, these results suggest that the object-representation systems of the ventral visual pathway are exploited for haptic object perception. (C) 2002 Elsevier Science Ltd. All rights reserved.
\end{abstract}

Keywords: fMRI; Priming; Somatosensory; Vision; Haptic; Object recognition; Neuroimaging; Human brain mapping

\section{Introduction}

Vision is the primary sensory modality that humans and other primates use to identify objects in their environment. Nevertheless, we also use our sense of touch (haptics) to perceive the shape, the size, and other characteristics of objects. In many cases, vision and haptics provide the same information about the object's structure and surface features. Although both systems can be used to identify objects, there are clear differences in the nature of the information that is immediately available to each system. The haptic system can receive information only about objects that reside within personal space, i.e. those objects that are within arm's reach. The visual system can receive information not only about objects that reside within personal space, but also those that are at some distance from the observer. When objects are at a distance, only the surfaces and parts of an object that face the observer can be processed visually. When objects

\footnotetext{
* Corresponding author. Tel.: +1-519-661-2070; fax: +1-519-661-3961. E-mail address: mgoodale@uwo.ca (M.A. Goodale).
}

are within reach, however, the object can be manipulated, thus, revealing the structure and features of the previously unseen surfaces and parts.

Despite the differences in the information that is available to vision and haptics, there is evidence that the higher-order processing of the two systems may deal with their respective inputs in much the same way. For example, in most situations, visual recognition of objects is viewpoint dependent. That is, if an object is visually explored from a particular viewing angle, recognition will be better for that view of the object than for other views [9,11]. Although the concept of 'viewing angle' in haptic exploration of fixed objects is not as well-defined as it is in vision, recent work by Newell et al. [16] has shown that haptic recognition of objects is much better when the study and test 'views' are the same. This suggests that the information about the structure of the object may be stored in a similar way by the visual and haptic systems.

In fact, there is some speculation that visual and haptic object representations are so similar that they might actually be shared between the two modalities. Reales and Ballesteros 
[19] used cross-modal priming between vision and haptics to show that exposure to real objects in one modality affected later naming of the objects when they were presented using the other modality. The term 'priming' refers to the facilitative effect that prior exposure to a stimulus has on the perception of that stimulus during a subsequent encounter. People are usually quite unaware of this facilitation. In a cross-modal experiment then, subjects would first be exposed to objects in one modality and then would be required to identify or discriminate between the same objects presented in the other modality. Interestingly, cross-modal priming and within-modality priming resulted in similar effect sizes, suggesting that activation of a haptic representation produces equal activation of a visual representation and vice versa. Although it is possible that this co-activation could be mediated by semantic or verbal representations of the objects, the fact that babies as young as 2 months of age, as well as chimpanzees [25], can perform cross-modal (visual-to-haptic) matching tasks, provides further evidence that visual and haptic object representations are shared between modalities. Finally, there is recent evidence to suggest that cross-modal recognition is viewpoint specific. In other words, an object studied haptically at one particular 'view' will be visually recognized better at that view than at other views [16]. Taken together, these studies suggest that visual and haptic representations overlap and that this overlap occurs at the level of three-dimensional shape representations, not at a more abstract level. In other words, the cross-modal representation depends more on the object's geometry than on its semantic labels and associations.

The behavioral evidence, then, suggests that vision and haptics represent the shape of objects in the same way. It is possible, therefore, that these two sensory systems could also share a common neural substrate for representing the shape of objects. Three studies suggest that the neural substrate underlying visual and haptic object recognition is found within extra-striate cortex. Two of these three studies [1,3] had subjects perform haptic object identification tasks while measuring brain activation using functional magnetic resonance imaging (fMRI). These investigators found that, compared to a control task, identifying objects haptically produced activation in extra-striate cortex, in addition to other regions. The third study [29] used transcranial magnetic simulation (TMS), a technique that produces a brief magnetic pulse that is intended to disrupt the processing occurring in a region of cortex. They applied TMS to different regions of cortex while subjects were asked to identify the orientation of a grating that was placed on their finger. When TMS was applied to the occipital cortex contralateral to the hand being used, subjects were not able to perform the task, but performed normally when TMS was applied to the ipsilateral occipital cortex.

The authors of two of the studies described earlier [3,29] concluded that visual imagery is invoked during haptic object recognition. Deibert et al. [3], who observed fMRI activation in occipital cortex during haptic exploration, argued that visual imagery is a byproduct of haptic processing that is not essential for recognition. Zangaladze et al. [29], however, who used TMS to disrupt occipital cortex, argued that visual imagery is necessary for successful haptic object recognition. The authors of the remaining fMRI study [1], while agreeing that visual imagery was invoked during haptic object recognition, did not believe that the activation in occipital cortex was entirely due to visual imagery. Amedi et al. [1] found that imagining objects resulted in much less activation in occipital cortex than haptically exploring them did. They concluded that some other mechanism besides visual imagery must be involved in activating the occipital cortex during haptic object recognition.

Although Amedi et al. [1] found that the region of occipital cortex that was activated during haptic object recognition did not show much activation for imagined objects, they did find that it responded strongly to the visual presentation of those objects. This region of occipital cortex, which is located in the ventral occipital cortex, has been found to respond preferentially to object stimuli in many other neuroimaging studies [8,12-15,18,23]. If, as Amedi et al. [1] suggest, the activation in ventral occipital cortex during haptic object processing is not due primarily to visual imagery, then perhaps it is due to the activation of a common neural substrate for visual and haptic object recognition. As suggested earlier, the idea of a common representation is supported by behavioral experiments that have studied cross-modal transfer between vision and haptics using priming paradigms $[4,5,19]$.

In the present study, we used a cross-modal priming method in combination with high-field fMRI to investigate the neural interactions between vision and haptics. It has been argued that cross-modal priming paradigms are a good tool for investigating the extent to which the representations

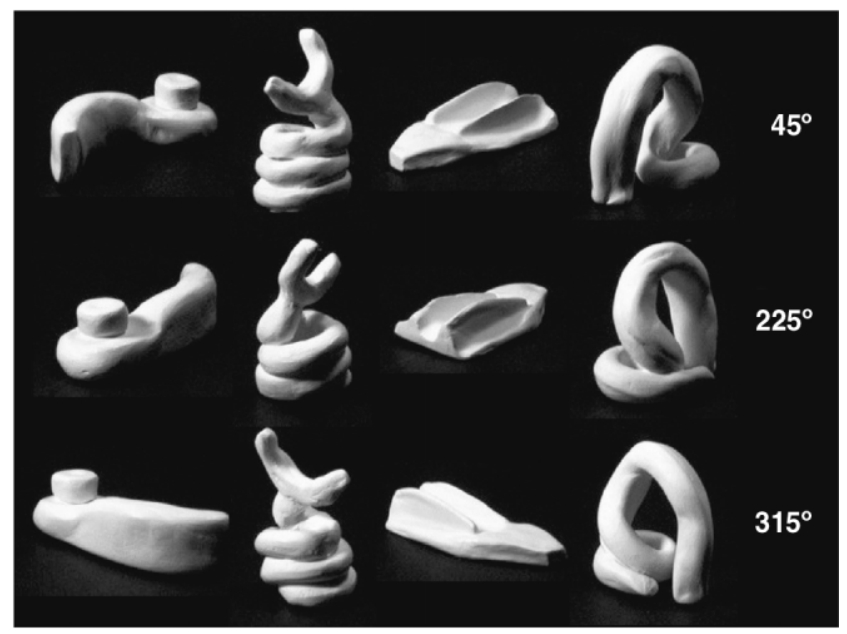

Fig. 1. Examples of novel three-dimensional clay objects. The images of the objects depicted here were taken from three different orientations relative to the camera's 'line of sight'. The 45 and $225^{\circ}$ views were used during the study phase. The 45 and $315^{\circ}$ views were used for the priming task portion of the test phase (see Section 2). 
of two different sensory modalities overlap [19]. Thus, we used a cross-modal priming paradigm to investigate whether or not there is a common neural substrate underlying the visual and haptic representations of object shape. Because we were interested in cross-modal representations of object shape, and consequently wished to minimize the possibility of semantic encoding or verbal labeling mediating any cross-modal priming, we used a set of three-dimensional novel objects made out of clay (Fig. 1). Previous priming studies that have used sets of novel objects have found discrepant results in terms of whether priming produces an increase or a decrease in brain activation $[10,21,27]$. Regardless of the direction of the priming effect in these different studies, the effect was always consistently in the same direction for each particular set of novel objects. Thus, our prediction was that the change in activation due to priming would be in the same direction regardless of whether the objects were studied visually or haptically.

\section{Materials and methods}

\subsection{Subjects}

Subjects were six right-handed graduate students attending the University of Western Ontario. All subjects reported normal or corrected-to-normal visual acuity and no known neurological or visual disorders. Ages ranged from 24 to 36 years with a mean age of 27.2 years. The ethical review boards of both the University of Western Ontario and the Robarts Research Institute approved a protocol for the procedure.

\subsection{Stimuli}

A set of 48 novel three-dimensional clay objects were selected from a larger, existing set that had been used in previous research $[9,11]$. All objects had a definable principal axis of elongation and were colored white. The length of this axis varied from 5.0 to $1.5 \mathrm{~cm}$. These clay objects were used during the study phase of the experiment. Images of each object were created by photographing the objects with a CCD camera interfaced to an Apple Macintosh computer. Images were acquired from three different viewpoints for each object, with the principal axis rotated clockwise 45, 225 or $315^{\circ}$ away from the line of sight of the camera (Fig. 1). Images were taken using a black background and were presented as grayscale bitmaps. When used during the testing phase of the experiment, no image subtended $<2.0$ or $>3.5^{\circ}$ of visual angle in the vertical or horizontal axes.

\subsection{Study phase procedure}

Subjects were seated at a table with a turntable $(10.0 \mathrm{~cm}$ diameter) on it. The center of the turntable was positioned $30.0 \mathrm{~cm}$ in front of them. Each object from the set of 48 objects was randomly assigned to one of three subsets of 16 objects, two subsets that were studied and one subset that was not studied. Objects were studied under two conditions, visual and haptic, and each subject participated in both study conditions. The subset of objects that was studied visually or haptically was counterbalanced across subjects. The non-studied subset of 16 objects was kept constant across subjects.

During the visual study condition, subjects were asked to close their eyes between trials while the experimenter placed or repositioned one of the clay objects on the turntable. To start the trial, the experimenter said "start" and the subject opened their eyes and viewed the object for $3 \mathrm{~s}$, at which time the experimenter said "stop" and the subject closed their eyes. This was repeated for each object for two different viewing angles. Each object was presented at both 45 and $225^{\circ}$ from the subject's line of sight. The view to be shown first was counterbalanced across objects and subjects.

During the haptic study condition, subjects closed their eyes for the entire study period. The experimenter used the same "start" and "stop" instructions that were used for the visual study condition, but "start" indicated that the subject begin palpating the object with both hands. Before beginning the study session, subjects were instructed that the object was not to be moved or picked up from the turntable and the objects were stable enough on the turntable that two-handed palpation did not move them significantly. Timing of the $3 \mathrm{~s}$ haptic study period was started as soon as the subject's hands contacted the object, which made the haptic study take slightly longer than the visual study. After each "stop" instruction, the experimenter either changed the presentation angle of the object or replaced it with the next object. During this time, the subjects held their hands away from the turntable so as not to interfere with the changing of the objects. The presentation angles of the objects (with respect to the subject's hypothetical line of sight) were the same as for the visual condition.

\subsection{Test phase procedure}

All imaging was done using a $4 \mathrm{~T}$, whole body MRI system (Varian/Siemens) and a quadrature head coil. The field of view was $19.2 \mathrm{~cm} \times 19.2 \mathrm{~cm} \times 6.6 \mathrm{~cm}$, with an in-plane resolution of $64 \times 64$ pixels and 11 contiguous coronal scan planes per volume, resulting in a voxel size of $3.0 \mathrm{~mm} \times 3.0 \mathrm{~mm} \times 6.0 \mathrm{~mm}$. Images were collected using a T2*-weighted, segmented (navigator corrected), interleaved EPI acquisition $(\mathrm{TE}=15 \mathrm{~ms}, \mathrm{TR}=500 \mathrm{~ms}$, flip angle $=$ $30^{\circ}, 2$ segments/plane) for blood-oxygen level dependent (BOLD) based imaging [17]. Each volume (11 planes) required $1.0 \mathrm{~s}$ to acquire and spanned a volume of cortex from the occipital pole to the splenium of the corpus collosum. High-resolution T1-weighted anatomical images were acquired using a three-dimensional magnetization prepared (MP) turbo FLASH acquisition with an inversion time (TI) of $600 \mathrm{~ms}\left(\mathrm{TE}=5.2 \mathrm{~ms}, \mathrm{TR}=10 \mathrm{~ms}\right.$, flip angle $\left.=15^{\circ}\right)$. 
Subjects lay in the magnet in a supine position with their head secured in the head coil with foam padding. Images were projected into the bore of the MRI system with an LCD projector and onto a rear-projection screen that straddled the subject's waist. Subjects were able to see the screen by looking into a mirror that was mounted within the head coil at $75 \mathrm{~cm}$ distance from the screen. Images of the objects were presented to the subjects using an event-related design. Each trial consisted of a $250 \mathrm{~ms}$ presentation of a red fixation cross, viewing of an image for 2.0 and a $12.0 \mathrm{~s}$ inter-trial interval during which subjects fixated a blue cross. The 45 and $315^{\circ}$ views of the objects were presented for the 16 visually studied objects, the 16 haptically studied objects, and the 16 objects that were not studied. Thus, for the primed objects, one of the views $\left(45^{\circ}\right)$ had been explicitly studied and the other view $\left(315^{\circ}\right)$ had not. This resulted in 96 trials that were presented in random order. Trials were conducted in four runs of 24 trials each lasting 350 s. In Section 3, these event-related runs will be called the priming task.

After the four event-related runs were completed, subjects viewed images of four different views of another set of eight novel objects that had not been studied. Thirty-two second periods of object viewing were interleaved with $32 \mathrm{~s}$ periods of fixation, with four object viewing periods and five fixation periods. Finally, subjects were asked to haptically explore two novel clay objects that were not part of any of the object sets described earlier. Again, $32 \mathrm{~s}$ periods of haptic exploration were interleaved with $32 \mathrm{~s}$ periods of fixation. During the exploration periods, subjects palpated one of the two objects with both hands for the entire $32 \mathrm{~s}$, while the other object rested on their abdomen. There were four exploration periods and five fixation periods. Because subjects had to receive the task instructions by visual cue, they were obliged to have their eyes open during haptic exploration; however, they were instructed to not bring the objects into their field of view during exploration. In Section 3, these tasks will be called the exploration tasks.

\section{Results}

The imaging data were analyzed using the Brain Voyager $^{\mathrm{TM}}$ three-dimensional analysis tools. Anatomical MRIs for each subject were transformed into a common brain space [26]. Functional MRIs for each subject were motion corrected on an individual slice basis and blurred in space and time using a Gaussian filter with a full-widthhalf-maximum of two pixels $(6 \mathrm{~mm})$ and two volumes $(2 \mathrm{~s})$, respectively. The functional images were then aligned to the transformed anatomical MRIs, thereby transforming the functional data into a common brain space and facilitating the comparison of data across subjects.

Data collected during the priming task were analyzed using the Brain Voyager ${ }^{\mathrm{TM}}$ multi-study GLM procedure. This procedure allows the correlation of predictor variables or functions with the recorded activation data (criterion variables) across scanning sessions. Primed objects were those objects that subjects studied either visually or haptically during the study phase of the priming experiment (see Section 2). Because the stimuli were all presented using the visual modality during the test phase, priming was either within-modal visual-to-visual priming or cross-modal
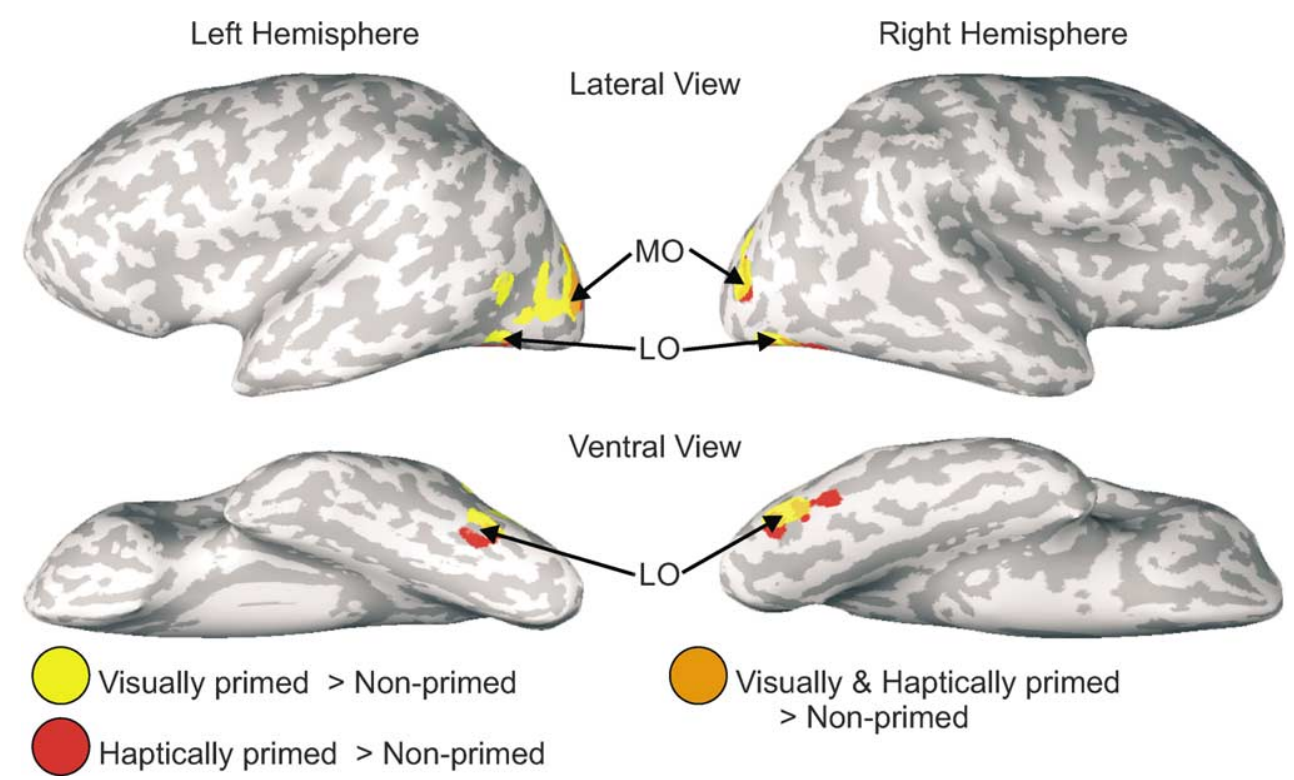

Fig. 2. Maps of the activation produced during the priming task. Brains are 'inflated' to allow activation within the sulci to be viewed. Gyri appear in light gray and sulci appear in dark gray. Lateral and medial views of both hemispheres are shown. Activation was seen in the middle occipital area (MO) and the lateral occipital area (LO). Yellow colored areas produced more activation with visually primed objects than with non-primed objects. Red colored areas produced more activation with haptically primed objects than with non-primed objects. Orange colored areas produced more activation with both visually and haptically primed objects than with non-primed objects. 

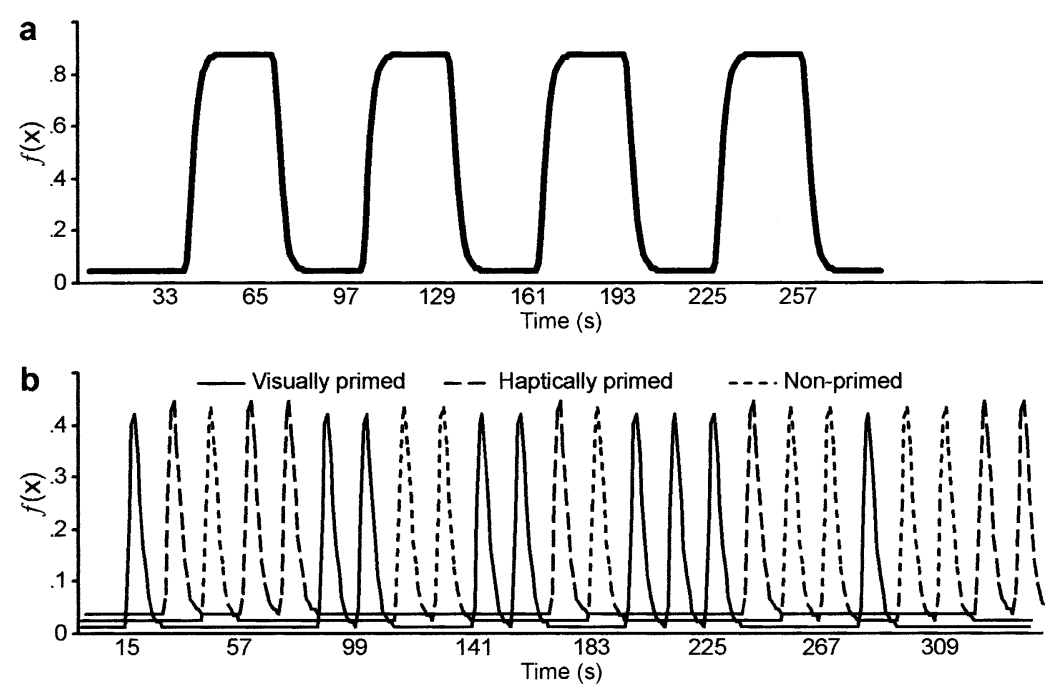

Fig. 3. Predictor functions used for the analysis of brain activation. (a) The predictor function for analyzing the data from both the visual and the haptic exploration tasks. The predictor function was made up of gamma functions $(\Delta=2.5, \tau=1.25)$ that were spaced apart in time based on the underlying structure of the blocked presentation of the stimuli. (b) The predictor functions for analyzing the data from the priming task. Each of the three predictor functions was made up of gamma functions $(\Delta=2.5, \tau=1.25)$ that were spaced apart in time based on the underlying structure of the individual, randomly ordered trials. There was one predictor function for visually primed object trials, one for haptically primed object trials, and one for non-primed object trials. Only 24 trials (the first 'run') of the 96 total trials are shown here. Predictor functions representing all 96 trials were used for the GLM analysis (see Section 3).

haptic-to-visual priming. The activation maps displayed in Fig. 2 show voxels that produced significantly greater activation $\left(\Delta R>0.1, P<10^{-7}\right)$ with visually or haptically primed objects than with non-primed objects. Significance was determined by examining the difference in the correlation of the recorded activation data from each voxel with a predictor function that was particular to each priming condition. Each of the three predictor functions was a series of 32 gamma functions ( $\Delta=2.5, \tau=1.25$ ) spaced in time based on the random stimulus presentation order (see Section 2). Fig. 3b illustrates an example of the three predictor functions for one of the four priming task runs. In this particular run, there were nine visually primed trials, seven haptically primed trials and eight non-primed trials. In Fig. 2, yellow colored areas were more activated by viewing visually primed when compared with non-primed objects and red areas were more activated by viewing haptically primed when compared with non-primed objects. Orange colored areas were more activated by viewing both visually primed and haptically primed objects when compared with non-primed objects.

Visually primed objects produced more activation than non-primed objects in the middle occipital (MO) and lateral occipital (LO) areas of the lateral occipital complex $[6,15]$. Haptically primed objects also produced more activation than non-primed objects in areas MO and LO. A complete description of these regions and their spatial coordinates is listed in Table 1. Fig. 4 illustrates the hemodynamic response function for areas LO and MO averaged across all 32 haptic, visual and non-primed trials and across all six subjects. As might be expected, considering the overlap of activation in areas MO and LO between the haptically and visually primed objects, the activation produced in these regions was equivalent for haptically and visually primed objects.

Data collected during the visual and haptic exploration tasks were also analyzed using the Brain Voyager $^{\mathrm{TM}}$ multi-study GLM procedure. The activation maps displayed in Fig. 5 show voxels (three-dimensional pixels) that produced significantly greater activation $\left(r>0.4, P<10^{-7}\right)$ during visual or haptic exploration than during fixation. Significance was determined by correlating the recorded

Table 1

Coordinates of regions modulated by visual and haptic priming

\begin{tabular}{lllll}
\hline Brain region & \multicolumn{3}{c}{ Talairach coordinates } & BA \\
\cline { 2 - 4 } & $X$ & $Y$ & $Z$ & \\
\hline Visual-to-visual priming & & & & \\
$\quad$ Middle occipital gyrus & 40 & -82 & +12 & 19 \\
Inferior occipital gyrus & 27 & -93 & -4 & 18 \\
Lingual gyrus & 12 & -93 & -7 & 17 \\
Middle occipital gyrus & 34 & -83 & -8 & 18 \\
Lingual gyrus & 13 & -93 & -12 & 17 \\
Fusiform gyrus & 36 & -51 & -12 & 37 \\
Fusiform gyrus & 23 & -83 & -12 & 19 \\
Haptic-to-visual priming & & & & \\
Fusiform gyrus & 36 & -69 & -12 & 19 \\
Fusiform gyrus & 40 & -51 & -16 & 37 \\
Visual and haptic priming & & & & \\
Middle occipital gyrus & 53 & -79 & +4 & 19 \\
Inferior temporal gyrus & 49 & -60 & -1 & 19 \\
Middle occipital gyrus & 47 & -75 & -4 & 19 \\
Middle occipital gyrus & 51 & -73 & -8 & \\
\hline
\end{tabular}




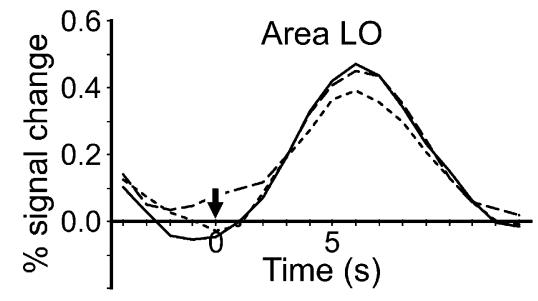

Visually primed

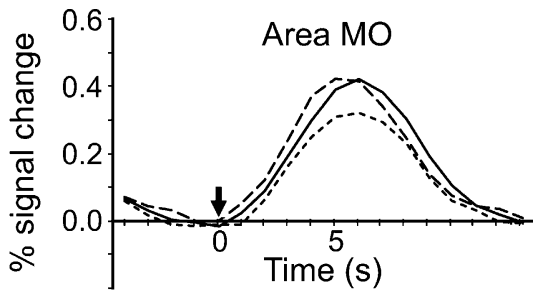

Non-primed

$\downarrow$ Stimulus onset

Fig. 4. Hemodynamic response curves for areas LO, MO. Activation is shown for visually primed, haptically primed, and non-primed objects averaged across 32 trials per condition and across all six subjects. Stimulus onset is indicated by the downward pointing arrow. Objects were presented for $2 \mathrm{~s}$, followed by $12 \mathrm{~s}$ of fixation.

activation data from each voxel with a predictor function shown in Fig. 3a that was a series of four gamma functions $(\Delta=2.5, \tau=1.25)$ spaced in time based on the blocked stimulus presentation paradigm (see Section 2). In Fig. 5, yellow colored areas were more activated during visual exploration than during fixation and red colored areas were more activated during haptic exploration than during fixation. Orange colored areas were more activated during both visual and haptic exploration than during fixation.
Visual exploration produced activation in the foveal representation of primary visual cortex (V1) and within the lateral occipital complex, including areas MO, LO and the fusiform gyrus (FG). Haptic exploration produced activation in primary somatosensory cortex (S1), an area of the central ventrolateral temporal lobe (CVLT; BA 20), area MO, the lingual gyrus (LG) and the peripheral representation of area V1. A complete description of these regions and their spatial coordinates is listed in Table 2.

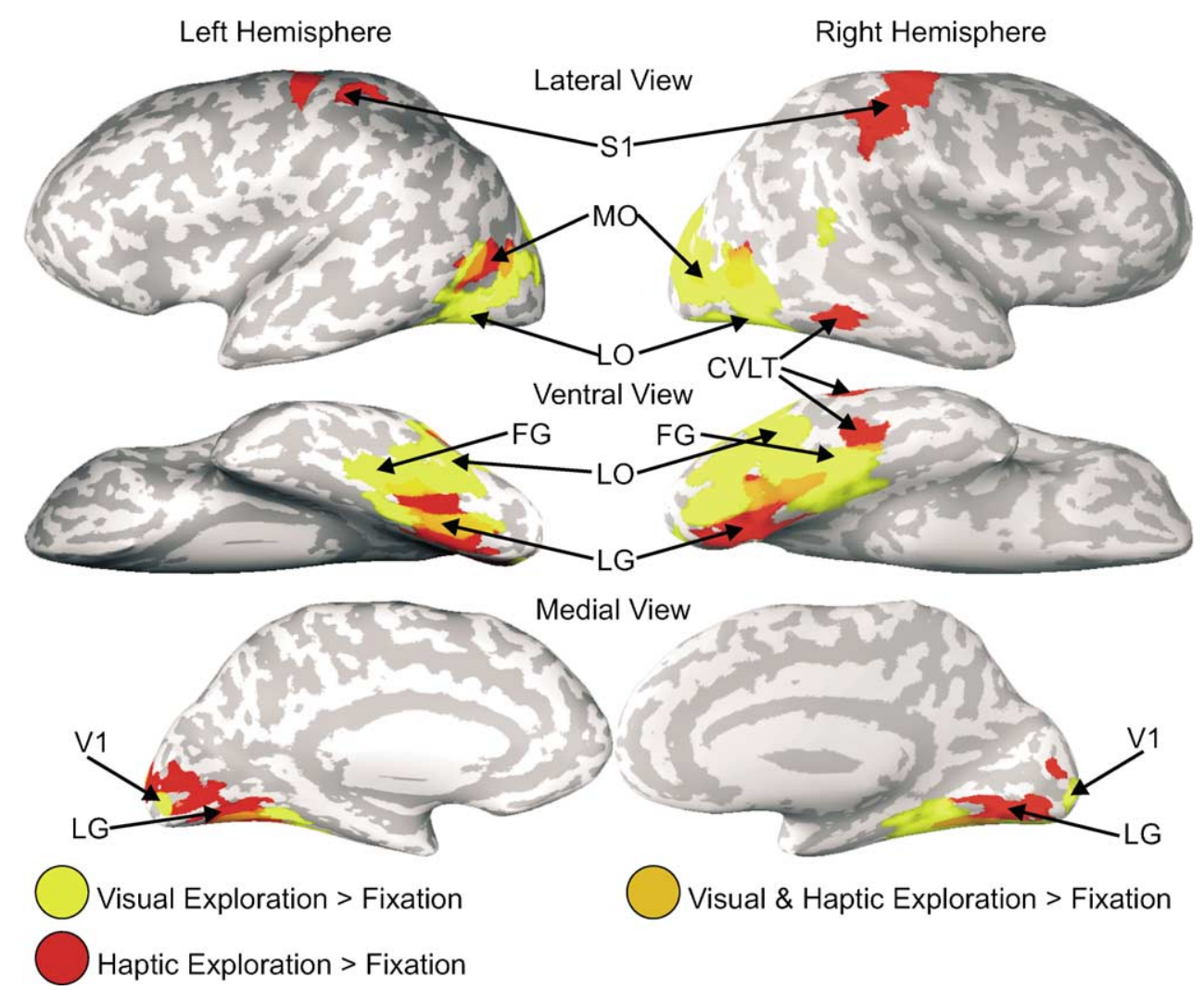

Fig. 5. Maps of the activation produced during the exploration tasks. Brains are 'inflated' to allow activation within the sulci to be viewed. Gyri appear in light gray and sulci appear in dark gray. Lateral, medial and ventral views of both hemispheres are shown. Activation was seen in primary visual cortex (V1), the lingual gyrus (LG), the middle occipital area (MO), the lateral occipital area (LO), the fusiform gyrus (FG), primary somatosensory cortex (S1) and the central ventrolateral temporal cortex (CVLT). Yellow colored areas produced more activation during visual exploration than during fixation. Red colored areas produced more activation during haptic exploration than during fixation. Orange colored areas produced more activation during both visual and haptic exploration than during fixation. 
Table 2

Coordinates of regions activated during visual and haptic exploration

\begin{tabular}{|c|c|c|c|c|}
\hline \multirow[t]{2}{*}{ Brain region } & \multicolumn{3}{|c|}{ Talairach coordinates } & \multirow[t]{2}{*}{ BA } \\
\hline & $X^{\mathrm{a}}$ & $Y$ & $Z$ & \\
\hline \multicolumn{5}{|l|}{ Visual } \\
\hline Superior temporal gyrus & +53 & -48 & +12 & 22 \\
\hline Middle occipital gyrus & 35 & -86 & +4 & 18 \\
\hline Parahippocampal gyrus & 36 & -43 & -4 & 19 \\
\hline Lingual gyrus & 3 & -93 & -4 & 17 \\
\hline Middle occipital gyrus & 53 & -61 & -4 & 19 \\
\hline Lingual gyrus & 21 & -77 & -8 & 18 \\
\hline Inferior occipital gyrus & 10 & -92 & -8 & 17 \\
\hline Fusiform gyrus & 36 & -69 & -12 & 19 \\
\hline Fusiform gyrus & 40 & -44 & -16 & 37 \\
\hline \multicolumn{5}{|l|}{ Haptic } \\
\hline Pre-central gyrus & 34 & -24 & +60 & 4 \\
\hline Post-central gyrus & 54 & -28 & +50 & 40 \\
\hline Post-central gyrus & 42 & -26 & +40 & 2 \\
\hline Lingual gyrus & 3 & -78 & +4 & 18 \\
\hline Lingual gyrus & 9 & -69 & -4 & - \\
\hline Middle temporal gyrus & +56 & -41 & -12 & 20 \\
\hline Fusiform gyrus & +43 & -34 & -20 & 20 \\
\hline \multicolumn{5}{|l|}{ Visual and haptic } \\
\hline Middle temporal gyrus & 44 & -63 & +4 & 37 \\
\hline Middle occipital gyrus & 32 & -85 & +4 & 18 \\
\hline Lingual gyrus & 24 & -54 & -8 & 19 \\
\hline
\end{tabular}

${ }^{a}$ A plus sign preceding the number indicates right hemisphere only.

\section{Discussion}

Haptic and visual exploration of novel three-dimensional clay objects produced activation in several different brain areas. Some brain regions showed activation during both kinds of exploration whereas others showed activation that was specific to either haptics or vision. Visual-to-visual and haptic-to-visual priming with the same objects increased the level of activation in a subset of these same areas, namely those that were activated by visual stimuli in the exploration phase. To our knowledge, the present study is the first to demonstrate an effect of haptic-to-visual cross-modal priming on brain activation. This cross-modal priming effect was observed in areas that are part of the lateral occipitotemporal complex (LOC), a region that has been previously implicated in the visual processing of objects $[8,12-15,18,23]$.

Like previous studies, the present study found that viewing objects produced activation in the LOC, including the middle and lateral occipital areas (MO and LO) and the fusiform gyrus (FG). The effects of cross-modal haptic-tovisual and within-modal visual-to-visual priming were observed in areas $\mathrm{MO}$ and LO, regions that have typically been thought to be involved in the visual processing of objects. Moreover, the magnitude of the increase in activation due to priming was the same in areas $\mathrm{MO}$ and $\mathrm{LO}$ regardless of whether the objects had been studied visually or haptically. Area FG, although activated during visual exploration, did not show differential activation with haptically and visually primed objects.
Although no behavioral data were collected in the present experiment, the fact that levels of activation were same for both kinds of priming parallels the results of a number of behavioral experiments $[4,5,19]$. In these studies, cross-modal priming effects between haptics and vision were quite similar in magnitude to the within-modal priming effects observed with either vision or haptics, even with novel objects. In both neural activation and behavior, then, cross-modal priming is no less 'efficient' in its effect than within-modal priming. Taken together, these findings suggest that no extra computational step is required to prime visual processing of object shape using a representation based on previous haptic input than is required to prime visual processing of shape using a representation based on previous visual input. Indeed, we would argue that cross-modal priming makes use of a common haptic and visual representation. One candidate region for the neural substrate of this common representation is area MO, which unlike area LO, was activated by both haptic and visual exploration, not only in our experiment but in others as well $[1,3]$.

As described in Section 1, however, Deibert et al. [3] suggested that haptic exploration of objects invokes visual imagery and this could account for the activation seen in the ventral occipital areas such as area MO. One report of a TMS experiment [29] has even suggested that visual imagery might be necessary for successful haptic recognition of objects. Does this mean that the activity we observed in area MO during haptic exploration (and during priming) is due to visual imagery? Although it is likely, as Deibert et al. in 2001 acknowledged that visual imagery often accompanies haptic exploration of objects, the evidence that it is necessary for recognition is not compelling. Easton et al. [4], e.g. have pointed out that haptic recognition of three-dimensional objects occurs so quickly that visual imagery could not be the mediating factor. Indeed, they concluded that haptic-to-visual cross-modal priming must depend on a common haptic and visual representation of object shape. Another interpretation of the Zangaladze et al. [29] result then, is that TMS applied to the occipital cortex had its effect on haptic recognition, not by interfering with visual imagery, but by disrupting the activity of the neural substrate that underlies this common representation.

But even if visual imagery is not necessary for haptic object recognition, it is still possible to argue, as Deibert et al. [3] did, that the activation seen in ventral occipital areas is largely due to visual imagery. Amedi et al. [1], however, tested this hypothesis directly by examining the response of the LOC during a visual imagery task. They showed that visually imagining objects produced much less activity in the LOC than did haptic exploration of the same objects. Thus, they concluded that the activation they recorded in the LOC during haptic exploration was not due to visual imagery, but reflected the activity of a multi-modal network. In fact, Amedi et al. [1] reported that the level of activation 
in the ventral occipital cortex during haptic exploration of objects was the same as during visual exploration of the same objects. In our study, area MO which was activated by both haptic and visual exploration, also showed equal levels of activation for both modalities. All of this suggests that the robust activation in area $\mathrm{MO}$ during haptic exploration is not due to visual imagery.

Not only did we find equivalent activation in area MO during haptic and visual exploration, but we also found equivalent activation in area MO as well area LO for both haptic-to-visual priming and visual-to-visual priming. For the same reasons outlined earlier, the equivalent activation during the priming tasks argues against a visual imagery account of cross-modal priming. Instead, as we argued earlier, the equivalent activation suggests that cross-modal priming depends on a common haptic and visual representation of object shape.

This common representation, we would argue, is not semantic or verbal in nature. As we stated in Section 1, we used novel objects instead of familiar objects to minimize the chances of semantic or verbal mediation of any priming effects that were observed. The fact that priming effects were found with these novel objects that are difficult to label verbally suggests that priming can occur below the level of semantic or verbal representations of objects. Thus, one might speculate that areas $\mathrm{MO}$ and LO are processing stimuli at the level of object shape.

The use of novel objects was probably also the reason why our priming effects showed an increase in activation instead of the usual decrease in activation that is seen in most priming studies (for review, see $[22,28]$ ). Two other studies that used novel objects $[10,21]$ also reported an increase in activation with primed objects as compared with non-primed objects. Why there should be this difference in the effects of priming with novel and familiar objects is not understood-and our study was not designed to address this question. We did, however, re-examine the priming task data at a lower statistical threshold $(t=1.0)$, and found no evidence for decreases in activation with primed objects relative to non-primed objects. It is also possible that, because we did not counterbalance the non-primed subset of objects, the increase in activation to the primed objects was due to an item effect. This explanation seems unlikely, because the objects were randomly assigned to the three subsets. Further, there were no obvious differences in the structure, complexity or other characteristics of the objects in the three subsets.

Many regions of the occipital cortex were activated during haptic exploration of objects in the present study. As was described earlier, the activation in the lateral occipital complex (of which MO is a part) during haptic object recognition has also been shown in two other neuroimaging studies [1,3]. In our study, however, several other occipital areas, including the lingual gyrus and striate cortex, were activated during haptic exploration that were not activated in these other studies $[1,3]$. This discrepancy in the pattern of activation may have been due to the fact that subjects in our experiment performed the haptic exploration task with their eyes open, whereas in the other two studies, subjects had their eyes closed. We tested our subjects with their eyes open to make the fixation control conditions during the visual exploration and haptic exploration tasks exactly the same and to facilitate comparisons between the activation produced during the visual and haptic exploration tasks. It is difficult to explain why having visual input during haptic exploration should lead to activation in these areas. The nature of the interaction between the presence of visual input (unrelated to the task) and haptic exploration remains to be explored.

Haptic exploration but not visual exploration also produced activation in the inferior temporal gyrus and the fusiform gyrus of the right central ventrolateral temporal lobe (CVLT; BA 20) in areas that are anterior to the well-established visual object processing areas. It is possible that these regions of the temporal lobe may be specialized for the processing of haptic information about the three-dimensional structure of objects. One might speculate further that these regions in area CVLT provide some of the input to area MO. There is some evidence from neuroimaging that regions within the insula and claustrum [2,7] and in the lateral parietal operculum $[20,24]$ also participate in the haptic recognition of objects. Thus, haptic exploration of objects may involve a network of brain regions that links input from primary somatosensory cortex to regions within the insula, parietal lobe and the ventral temporal lobe. This network might then share connections with many of the putative visual object processing regions in the occipital lobe such as area MO.

In summary, we have shown that haptic exploration of novel three-dimensional objects produces activation, not only in somatosensory cortex, but also in areas of the occipital cortex associated with visual processing. Furthermore, previous haptic experience with these same objects enhanced activation in visual areas when they were subsequently viewed. The striking overlap in some of the neural substrates mediating haptic and visual processing of object structure suggests that the haptic system may exploit the highly developed object representation systems of the ventral visual pathway.

\section{Acknowledgements}

The research was supported by grants from the Natural Sciences and Engineering Research Council of Canada, the Canada Research Chairs Program, and the Canadian Institutes of Health Research. Thanks to Karin H. James, Stefan Kohler, Susanne Ferber and two anonymous reviewers for their helpful suggestions on an earlier version of this manuscript. Thanks to Joseph F.X. Desouza for the discussions about the labeling of functional brain regions in Figs. 2 and 5. 


\section{References}

[1] Amedi A, Malach R, Hendler T, Peled S, Zohary E. Visuo-haptic object-related activation in the ventral visual pathway. Nature Neuroscience 2001;4:324-30.

[2] Bonda E, Petrides M, Evans A. Neural systems for tactual memories. Journal of Neurophysiology 1996;75:1730-7.

[3] Deibert E, Kraut M, Kremen S, Hart JJ. Neural pathways in tactile object recognition. Neurology 1999;52:1413-7.

[4] Easton RD, Greene AJ, Srinivas K. Transfer between vision and haptics: memory for 2-D patterns and 3-D objects. Psychonomic Bulletin and Review 1997;4:403-10.

[5] Easton RD, Srinivas K, Greene AJ. Do vision and haptics share common representations? Implicit and explicit memory within and between modalities. Journal of Experimental Psychology: Learning, Memory, and Cognition 1997;23:153-63.

[6] Grill-Spector K, Kushnir T, Hendler T, Edelman S, Itzchak Y, Malach R. A sequence of object-processing stages revealed by fMRI in the human occipital lobe. Human Brain Mapping 1998;6:31628.

[7] Hadjikhani N, Roland PE. Cross-modal transfer of information between the tactile and the visual representations in the human brain: a positron emission tomographic study. The Journal of Neuroscience 1998; 18:1072-84.

[8] Halgren E, Dale AM, Sereno MI, Tootell RBH, Marinkovic $\mathrm{K}$, Rosen BR. Location of human face-selective cortex with respect to retinotopic areas. Human Brain Mapping 1999;7:2937.

[9] Harman KL, Humphrey GK. Encoding 'regular' and 'random' sequences of views of novel three-dimensional objects. Perception 1999;28:601-15.

[10] Henson R, Shallice T, Dolan R. Neuroimaging evidence for dissociable forms of repetition priming. Science 2000;287:1269_ 72.

[11] Humphrey GK, Khan SC. Recognizing novel views of threedimensional objects. Canadian Journal of Psychology 1992;46: 170-90.

[12] James TW, Humphrey GK, Gati JS, Menon RS, Goodale MA. The effects of visual object priming on brain activation before and after recognition. Current Biology 2000;10:1017-24.

[13] Kanwisher N, Chun MM, McDermott J, Ledden PJ. Functional imaging of human visual recognition. Cognitive Brain Research 1996;5:55-67.

[14] Kraut M, Hart JJ, Soher BJ, Gordon B. Object shape processing in the visual system evaluated using fMRI. Neurology 1997;48:141620 .
[15] Malach R, Reppas JB, Benson RR, Kwong KK, Jiang H, Kennedy WA, et al. Object-related activity revealed by functional magnetic resonance imaging in human occipital cortex. Proceedings of the National Academy of Science 1995;92:8135-9.

[16] Newell FN, Ernst MO, Tjan BS, Bulthoff HH. Viewpoint dependence in visual and haptic object recognition. Psychological Science 2001;12:37-42.

[17] Ogawa S, Menon RS, Tank DW, Kim S-G, Merkle H, Ellermann JM. Functional brain mapping by blood oxygenation level-dependent contrast magnetic resonance imaging: a comparison of signal characteristics with a biophysical model. Biophysical Journal 1993;64: 803-12.

[18] Price CJ, Moore CJ, Humphreys GW, Frackowiak RSJ, Friston KJ. The neural regions sustaining object recognition and naming. Proceedings of the Royal Society of London Series B 1996;263: 1501-7.

[19] Reales JM, Ballesteros S. Implicit and explicit memory for visual and haptic objects: cross-modal priming depends on structural descriptions. Journal of Experimental Psychology: Learning, Memory, and Cognition 1999;25:644-63.

[20] Roland PE, O'Sullivan B, Kawashima R. Shape and roughness activate different somatosensory areas in the human brain. Proceedings of the National Academy of Science 1998;95:3295-300.

[21] Schacter DL, Reiman E, Uecker A, Polster MR, Yun LS, Cooper LA. Brain regions associated with retrieval of structurally coherent visual information. Nature 1995;376:587-90.

[22] Schacter DL, Buckner RL. Priming and the brain. Neuron 1998;20:185-95.

[23] Sergent J, Ohta S, Macdonald B. Functional neuroanatomy of face and object processing: a positron emission tomography study. Brain 1992;115:15-36.

[24] Servos P, Lederman SJ, Wilson D, Gati JS. fMRI-derived cortical maps for haptic shape, texture, and hardness. Cognitive Brain Research 2001;12:307-13

[25] Streri A. Seeing, reaching, touching: the relations between vision and touch in infancy. Cambridge, MA: MIT Press, 1993.

[26] Talairach J, Tournoux P. Co-planar sterotaxic atlas of the human brain. New York: Thieme Georg, Stuttgart, 1988.

[27] van Turennout M, Ellmore T, Martin A. Long-lasting cortical plasticity in the object naming system. Nature Neuroscience 2000;3:1329-34

[28] Wiggs CL, Martin A. Properties and mechanisms of perceptual priming. Current Opinion in Neurobiology 1998;8:227-33.

[29] Zangaladze A, Epstein CM, Grafton ST, Sathian K. Involvement of visual cortex in tactile discrimination of orientation. Nature 1999;401:587-90. 